\title{
Pharmacokinetics of glycopyrronium/formoterol fumarate dihydrate delivered via metered dose inhaler using co-suspension delivery technology in patients with moderate-to-very severe COPD
}

This article was published in the following Dove Press journal:

International Journal of COPD

\author{
Gary T Ferguson' \\ Roberto Rodriguez-Roisin ${ }^{2}$ \\ Colin Reisner ${ }^{3,4}$ \\ Andrea Maes ${ }^{3}$ \\ Shahid Siddiqui ${ }^{4}$ \\ Ubaldo J Martin ${ }^{4}$ \\ 'Pulmonary Research Institute of \\ Southeast Michigan, Farmington Hills, \\ MI, USA; ${ }^{2}$ Universitat de Barcelona, \\ Hospital Clínic-The August Pi i \\ Sunyer Biomedical Research Institute \\ (IDIBAPS), Barcelona, Spain; ${ }^{3}$ Pearl - A \\ member of the AstraZeneca Group, \\ Morristown, NJ, USA; ${ }^{4}$ AstraZeneca, \\ Gaithersburg, MD, USA
}

\begin{abstract}
Purpose: The efficacy and tolerability of GFF MDI (Bevespi Aerosphere ${ }^{\circledR}$ ), a fixed-dose combination of glycopyrronium (GP)/formoterol fumarate dihydrate (FF) 14.4/10 $\mu \mathrm{g}$ (equivalent to glycopyrrolate/formoterol fumarate $18 / 9.6 \mu \mathrm{g}$ ) delivered by metered dose inhaler (MDI) using innovative co-suspension delivery technology, has been investigated in a Phase III clinical trial program (NCT01854645, NCT01854658, NCT01970878) in patients with COPD. Here, we present findings from a pharmacokinetic (PK) sub-study of NCT01854645 (PINNACLE-1).
\end{abstract}

Methods: PINNACLE-1 was a multicenter, randomized, double-blind, parallel-group, 24 wk chronic-dosing, placebo- and active-controlled study. The PK sub-study assessed the systemic accumulation of glycopyrronium and formoterol following administration of GFF MDI 14.4/10 $\mu \mathrm{g}$, GP MDI $14.4 \mu \mathrm{g}$, or FF MDI $10 \mu \mathrm{g}$ (all BID) for 12 wks. Plasma for PK analysis was collected for up to $12 \mathrm{~h}$ after dosing, on Day 1 and Week 12.

Results: Of 2,103 patients randomized in PINNACLE-1, 292 participated in the PK sub-study. The plasma concentration-time profiles of glycopyrronium were similar following treatment with GFF MDI or GP MDI, both after single dosing and at Week 12. Accumulation at Week 12 relative to Day 1 was up to 2.30-fold for glycopyrronium. The plasma concentration-time profiles of formoterol were similar following treatment with GFF MDI or FF MDI, both after single dosing and at Week 12. Accumulation at Week 12 relative to Day 1 was up to 1.62-fold for formoterol.

Conclusion: Overall, the results have characterized the accumulation of glycopyrronium and formoterol associated with GFF MDI, GP MDI, and FF MDI, and indicated that there were no meaningful PK interactions, whether drug-drug or due to formulation, between glycopyrronium and formoterol following treatment with GFF MDI formulated using co-suspension delivery technology.

Keywords: COPD, glycopyrronium, formoterol fumarate dihydrate, metered dose inhaler, co-suspension delivery technology, pharmacokinetics

\section{Introduction}

Monotherapy or dual therapy with long-acting muscarinic antagonist (LAMA) and long-acting $\beta_{2}$-agonist (LABA) bronchodilators is central to the maintenance treatment of COPD. ${ }^{1}$ Combined LAMA/LABA therapy has been shown to improve bronchodilation to a greater degree than LAMAs or LABAs alone, with a similar safety profile. ${ }^{2-6}$ Delivering dual LAMA/LABA therapy as a fixed-dose combination (FDC) in a single device may improve adherence and, therefore, also improve outcomes compared with multiple devices. ${ }^{7,8}$
Correspondence: Gary T Ferguson Pulmonary Research Institute of Southeast Michigan, 29255 W 10 Mile Rd a, Farmington Hills, MI 48336, USA

Tel +l 248478656 I

Email garytferguson@msn.com 
The dual bronchodilator therapy glycopyrronium/ formoterol fumarate dihydrate (GFF) is a LAMA/LABA FDC delivered by metered dose inhaler (MDI) using innovative co-suspension delivery technology. GFF MDI (Bevespi Aerosphere $^{\circledR}$; AstraZeneca Pharmaceuticals LP, Wilmington, DE, USA) $14.4 / 10 \mu \mathrm{g}$ (equivalent to glycopyrrolate/ formoterol fumarate $18 / 9.6 \mu \mathrm{g}$ ), has been approved in the USA for the long-term maintenance treatment of airflow obstruction in patients with COPD. ${ }^{9}$ The co-suspension delivery technology used to formulate glycopyrronium (GP) and formoterol fumarate dihydrate (FF) together in GFF MDI enables the uniform delivery of both treatments. ${ }^{10-12}$

Two pivotal 24 wk, Phase III studies of GFF MDI (PINNACLE-1 [NCT01854645] and PINNACLE-2 [NCT01854658]) demonstrated that GFF MDI had a similar safety profile to monocomponent MDIs (GP MDI and FF MDI, delivered using co-suspension delivery technology), placebo MDI, and open-label tiotropium. ${ }^{4}$ Additionally, GFF MDI showed superior efficacy to monocomponent MDIs and placebo in patients with moderate-to-very severe COPD. ${ }^{4}$ A 28 wk safety extension of these studies (PINNACLE-3 [NCT01970878]) confirmed the similar safety profile of GFF MDI to monocomponent MDIs and open-label tiotropium over 52 wks. ${ }^{13}$ GFF MDI also maintained the significant improvements in lung function end points compared with monocomponent MDIs over 52 wks. ${ }^{13}$

The pharmacokinetic (PK) profile of GFF MDI 28.8/10 $\mu \mathrm{g}$ compared with monocomponent MDIs, GP MDI $28.8 \mu \mathrm{g}$, and FF MDI $10 \mu \mathrm{g}$ has previously been described in a Phase IIb study in patients with moderate-to-very severe COPD after $7 \mathrm{~d}$ of chronic dosing. ${ }^{14}$ Here we present findings from a PK sub-study of PINNACLE-1, which assessed the systemic accumulation of glycopyrronium and formoterol and evidence for PK interaction following administration of GFF MDI 14.4/10 $\mu \mathrm{g}$ versus the monocomponent MDIs, GP MDI $14.4 \mu \mathrm{g}$, and FF MDI $10 \mu \mathrm{g}$, for 12 wks in patients with moderate-to-very severe COPD.

\section{Methods}

\section{Study design}

This PK assessment was conducted as a sub-study of PINNACLE-1, a multicenter, randomized, double-blind, parallel-group study to assess the efficacy and safety of GFF MDI 14.4/10 $\mu \mathrm{g}$, GP MDI $14.4 \mu \mathrm{g}$, and FF MDI $10 \mu \mathrm{g}$ compared with placebo MDI (all BID), and open-label tiotropium bromide once daily in patients with moderate-tovery severe COPD over 24 wks. ${ }^{4}$ The PK assessments were obtained from a subset of patients randomized to each active treatment arm, excluding open-label tiotropium, but including patients randomized to placebo MDI. However, the patients in the PK sub-study who received placebo MDI were not included in the PK population for analysis.

The primary PK objective was to assess the systemic accumulation of glycopyrronium and formoterol following BID administration of GFF MDI, GP MDI, and FF MDI for 12 wks. An additional objective was to evaluate whether evidence for PK interactions was present when glycopyrronium and formoterol are formulated together in GFF MDI.

\section{Study patients}

Patients in the PINNACLE-1 study were current or former smokers ( $\geq 10$ pack-years), 40-80 years of age, who had a clinical history of moderate-to-very severe COPD. Key inclusion and exclusion criteria of PINNACLE-1 have been previously published. ${ }^{4}$ For inclusion in the PK population, patients had to have sufficient plasma concentration data to calculate at least one PK parameter on either Day 1 or at Week 12 and no major protocol deviations that affected the collection and interpretation of PK data.

\section{Sample collection and assessments}

Plasma samples were collected from $\sim 5 \mathrm{~mL}$ of whole blood by direct venipuncture (or from an indwelling intravenous cannula) $30 \mathrm{~min}$ prior to dosing and at 2, 5, and $20 \mathrm{~min}$, and $1,2,4,8,10$, and $12 \mathrm{~h}$ after dosing, on Day 1 (after a single dose) and at Week 12 (after chronic dosing).

Plasma concentrations of glycopyrronium and formoterol were determined by Tandem Labs (Salt Lake City, UT, USA) using a validated high-performance liquid chromatography tandem mass spectrometry method.

\section{PK and statistical analysis}

Prior to the final PK analysis, patient data (eg, concomitant medications, dosing information from the patient diary, and adverse events and protocol deviations) were reviewed in a blinded manner by Everest Clinical Research Services, Inc. (Markham, ON, Canada) and Pearl - A member of the AstraZeneca Group (Redwood City, CA, USA) for inclusion/ exclusion into the PK population.

Patients who had missed one or more doses of study medication in the $2 \mathrm{~d}$ prior to the Week 12 visit were excluded from the analysis of Week 12 data since these patients were not at steady state. In addition, the $12 \mathrm{~h}$ plasma concentration value at Week 12 was excluded if patients took their evening dose of study medication prior to the collection of the $12 \mathrm{~h}$ blood sample. 


\section{PK parameters}

Mean plasma concentration-time profiles for glycopyrronium and formoterol were plotted, and descriptive statistics were calculated for plasma concentration data at each nominal time point. For presentation of these data, all values below the lower limit of quantification (LLQ) were assigned a value of $1 / 2$ LLQ, with the exception of Day 1 pre-dose values, which were assigned a value of zero and no geometric mean was calculated. The LLQ for formoterol was $1.0 \mathrm{pg} / \mathrm{mL}$. The LLQ for glycopyrronium was $2.0 \mathrm{pg} / \mathrm{mL}$ initially but was lowered to $1.0 \mathrm{pg} / \mathrm{mL}$ during the study.

Individual plasma glycopyrronium and formoterol concentration-time profiles were plotted for GFF MDI, GP MDI, and FF MDI at Day 1 and Week 12. $\mathrm{AUC}_{0-12}, \mathrm{C}_{\max }$, and $t_{\text {max }}$ were estimated for each patient and were calculated by non-compartmental analysis (NCA) using the software Phoenix ${ }^{\circledR}$ WinNonlin ${ }^{\circledR}$ (Pharsight Corporation, Mountain View, CA, USA). For the purposes of parameter estimation, post-dose plasma concentration values below the LLQ were set to "missing" in the NCA. Pre-dose concentration values below the LLQ were set to "zero".

\section{Systemic accumulation}

Systemic accumulations of glycopyrronium and formoterol were evaluated using natural-log transformed $\mathrm{AUC}_{0-12}$ and $\mathrm{C}_{\max }$, which were analyzed using a mixed model with a fixed effect (visit) and a random effect (patient). For each treatment, the estimated ratio of geometric least squares mean (LSM) for Week 12 to Day 1 and the corresponding 90\% CI were determined by exponentiating the mean difference between Week 12 and Day 1 and the associated 90\% CI (on the logarithm scale). The estimated ratio of geometric LSM with $90 \% \mathrm{CI}$ was an estimate of the accumulation ratio with $90 \% \mathrm{CI}$. To provide formulation-specific estimates of intra-subject variability, a separate mixed model was fit for each treatment arm.

\section{Relative bioavailability}

The relative bioavailability of glycopyrronium and formoterol (expressed as a ratio of the exponentiated mean difference of the log-transformed PK parameter for GFF MDI relative to GP MDI and FF MDI, respectively) at Day 1 and Week 12 was evaluated using a separate mixed model with fixed effects (treatment, visit, and treatment-by-visit interaction) and a random effect (patient). Since relative bioavailability among formulations was based on between-patient information, demographic and baseline covariates were evaluated for inclusion in the model using backward elimination. For glycopyrronium, additional covariates used in the final $\mathrm{AUC}_{0-12}$ model were race (Caucasian, Black, other), weight, height, and smoking status, and in the final $\mathrm{C}_{\max }$ model were race (Caucasian, Black, other), age, inhaled corticosteroid use, weight, and smoking status. For formoterol, additional covariates used in the final $\mathrm{AUC}_{0-12}$ model were age, weight, and height, and in the final $\mathrm{C}_{\text {max }}$ model were race (Caucasian, Black, other), weight, and height. For each visit, the ratios of geometric LSM and the corresponding 90\% CI for GFF MDI relative to GP MDI and FF MDI were determined by exponentiating the mean differences between treatments and $90 \% \mathrm{CI}$ on the logarithm scale. Also, because of between-patient assessment, high variability was anticipated and bounds for equivalence evaluation were not pre-specified.

\section{Ethics approval and informed consent}

This study was conducted in accordance with Good Clinical Practice Guidelines including the International Conference on Harmonisation, the US Code of Federal Regulations, and the Declaration of Helsinki. Institutional review boards (Schulman Associates IRB, Cincinnati, OH, USA, and Schulman Associates IRB, Ft Lauderdale, FL, USA) approved the protocol and informed consent form, and written informed consent was obtained from patients prior to screening.

\section{Results}

\section{Study population}

Of the 2103 patients who were randomized in the PINNACLE-1 study, 292 patients participated in the PK sub-study. There were no meaningful differences in baseline demographics and clinical characteristics of the patients in the PK sub-study across treatment groups (Table 1). The majority of patients were Caucasian $(92.8 \%)$ and male (57.5\%) from sites in Australia, New Zealand, and the USA. The mean age was 60.7 years and the mean body mass index was $28.9 \mathrm{~kg} / \mathrm{m}^{2}$. A total of 221 patients were included in the PK population; of those, 83 received GFF MDI, 70 received FF MDI, and 68 received GP MDI.

\section{Plasma concentrations and PK parameters}

The plasma concentration-time profiles of glycopyrronium following treatment with GFF MDI and GP MDI had similar profiles on Day 1 (after a single dose) and at Week 12 (after chronic dosing) (Figure 1A), with a median $\mathrm{t}_{\max }$ of $0.083 \mathrm{~h}$ (ie, $5 \mathrm{~min}$ ) post-dose irrespective of treatment (GFF MDI or GP MDI) or sample day (Table 2).

The plasma concentration-time profiles of formoterol following treatment with GFF MDI and FF MDI had similar profiles on Day 1 and at Week 12 (Figure 1B). The median 
Table I Baseline demographics and clinical characteristics (PK sub-study)

\begin{tabular}{|c|c|c|c|c|c|}
\hline Patient characteristics & $\begin{array}{l}\text { GFF MDI } \\
\text { I4.4/ I0 } 0 \mathrm{~g} \\
\mathrm{~N}=9 \text { | }\end{array}$ & $\begin{array}{l}\text { GP MDI } \\
\text { I } 4.4 \mu \mathrm{g} \\
\mathrm{N}=79\end{array}$ & $\begin{array}{l}\text { FF MDI } \\
10 \mu \mathrm{g} \\
\mathrm{N}=80\end{array}$ & $\begin{array}{l}\text { Placebo } \\
\text { MDI } \\
\mathbf{N}=\mathbf{4 2}\end{array}$ & $\begin{array}{l}\text { All patients } \\
\mathrm{N}=\mathbf{2 9 2}\end{array}$ \\
\hline Mean age, years (SD) & $60.4(7.9)$ & $61.6(8.7)$ & $60.5(8.8)$ & $59.9(9.0)$ & $60.7(8.5)$ \\
\hline Male, n (\%) & $54(59.3)$ & $45(57.0)$ & $43(53.8)$ & $26(61.9)$ & $168(57.5)$ \\
\hline Mean BMI, kg/m² (SD) & $28.8(5.9)$ & $29.8(6.4)$ & $28.5(6.6)$ & $28.3(5.2)$ & $28.9(6.1)$ \\
\hline Caucasian, n (\%) & $87(95.6)$ & $69(87.3)$ & $75(93.8)$ & $40(95.2)$ & $271(92.8)$ \\
\hline Current smoker, ${ }^{a}$ n (\%) & $57(62.6)$ & $50(63.3)$ & $48(60.0)$ & $28(66.7)$ & I $83(62.7)$ \\
\hline Mean pack-years ${ }^{\mathrm{b}}(\mathrm{SD})$ & $55.3(34.1)$ & $53.8(29.6)$ & $53.8(28.8)$ & $58.3(35.7)$ & $54.9(31.7)$ \\
\hline ICS at baseline, $n$ (\%) & $30(33.0)$ & $26(32.9)$ & $22(27.5)$ & $14(33.3)$ & $92(31.5)$ \\
\hline Mean CAT score ${ }^{c, d}(S D)$ & $19.9(6.9)$ & $20.3(7.7)$ & $19.5(7.4)$ & $20.0(6.5)$ & $19.9(7.2)$ \\
\hline Mean mMRC grade $(\mathrm{SD})$ & $\mathrm{I} .8(\mathrm{I} .0)$ & $2.0(1.1)$ & $2.1(1.0)$ & $2.0(I .1)$ & $2.0(1.0)$ \\
\hline
\end{tabular}

Notes: ${ }^{a}$ Former smoker was defined as those who have stopped smoking for at least 6 wks prior to first screening visit. ${ }^{b}$ Number of pack-years smoked $=($ number of

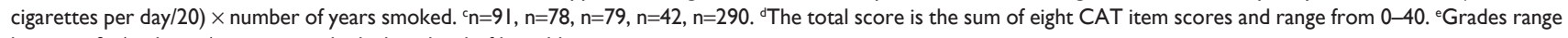
between $0-4$, where 4 represents the highest level of breathlessness.

Abbreviations: PK, pharmacokinetic; GFF, glycopyrronium/formoterol fumarate dihydrate; MDI, metered dose inhaler; GP, glycopyrronium; FF, formoterol fumarate dihydrate; BMI, body mass index; ICS, inhaled corticosteroid; CAT, COPD Assessment Test; mMRC, modified Medical Research Council dyspnea scale.

$\mathrm{t}_{\max }$ was numerically earlier for patients receiving GFF MDI (median $\mathrm{t}_{\max }: 0.41 \mathrm{~h}$ and $0.37 \mathrm{~h}$ on Day 1 and at Week 12 , respectively) than for patients receiving FF MDI (median $\mathrm{t}_{\max }$ : $1.00 \mathrm{~h}$ and $0.97 \mathrm{~h}$ for Day 1 and at Week 12, respectively) (Table 2). However, the mean $\mathrm{t}_{\max }$ was generally comparable for patients receiving GFF MDI (mean $\mathrm{t}_{\max }[\mathrm{CV} \%]: 0.92 \mathrm{~h}$ [102\%] and $0.92 \mathrm{~h}[137 \%]$ on Day 1 and at Week 12, respectively), compared with patients receiving FF MDI (mean $\mathrm{t}_{\text {max }}$ [CV\%]: $1.24 \mathrm{~h}$ [133\%] and $1.06 \mathrm{~h}$ [106\%] on Day 1 and at Week 12, respectively).

\section{Systemic accumulation of glycopyrronium and formoterol}

After 12 wks of chronic dosing, the glycopyrronium accumulation ratios for $\mathrm{AUC}_{0-12}$ were 2.30 for GFF MDI and 1.99 for GP MDI (Table 3). The glycopyrronium accumulation ratios for $\mathrm{C}_{\max }$ were 1.40 for GFF MDI and 1.26 for GP MDI at Week 12 (Table 3). After 12 wks of chronic dosing, the formoterol accumulation ratios for $\mathrm{AUC}_{0-12}$ were 1.52 for GFF MDI and 1.62 for FF MDI (Table 3). The formoterol accumulation ratios for $\mathrm{C}_{\max }$ were 1.32 for GFF MDI and 1.51 for FF MDI at Week 12 (Table 3). The intra-subject coefficients of variation were high for both glycopyrronium and formoterol (ranging from $30.1 \%$ to $54.9 \%$ and $26.4 \%$ to $38.1 \%$, respectively; Table 3 ).

\section{Relative bioavailability of glycopyrronium and formoterol}

The relative bioavailability for glycopyrronium $\mathrm{AUC}_{0-12}$ (GFF MDI to GP MDI) was 94.25\% (90\% CI: 78.52, 113.14) on Day 1 and $100.60 \%(90 \%$ CI: $84.23,120.14)$ at Week 12 . The relative bioavailability for glycopyrronium $\mathrm{C}_{\text {max }}$ (GFF MDI to GP MDI) was $100.92 \%(90 \%$ CI: 83.00, 122.72) on Day 1 and $109.10 \%$ (90\% CI: 88.87, 133.92) at Week 12 (Figure 2).
The relative bioavailability for formoterol $\mathrm{AUC}_{0-12}$ (GFF MDI to FF MDI) was 112.11\% (90\% CI: 99.33, 126.52) on Day 1 and $100.91 \%$ (90\% CI: 88.37, 115.24) at Week 12 . The relative bioavailability for formoterol $\mathrm{C}_{\max }$ (GFF MDI to FF MDI) was $118.76 \%$ (90\% CI: 104.87 , 134.50) on Day 1 and $101.33 \%$ (90\% CI: 88.60, 115.89) at Week 12 (Figure 2).

\section{Discussion}

The primary objective of this PK sub-study of a randomized, placebo-controlled Phase III study was to characterize the systemic accumulation of glycopyrronium and formoterol in patients receiving GFF MDI, or the individual monocomponents GP MDI and FF MDI, delivered via innovative co-suspension delivery technology. This study also provided a comparison of PK parameters for glycopyrronium between GFF MDI and GP MDI, and for formoterol between GFF MDI and FF MDI.

After a single dose and 12 wks of chronic dosing, the $12 \mathrm{~h}$ glycopyrronium plasma time-concentration profiles in patients receiving GFF MDI and GP MDI were comparable. Likewise, the formoterol plasma time-concentration profiles were comparable for patients receiving GFF MDI and FF MDI at both time points. For $\mathrm{AUC}_{0-12}$, up to a 2.30-fold accumulation for glycopyrronium following BID dosing (weak-to-moderate) and up to a 1.62-fold accumulation for formoterol following BID dosing (weak) were observed at Week 12 relative to Day $1 .{ }^{15}$ These findings are comparable with a previous study that determined that the steady-state accumulation ratio of $\mathrm{AUC}_{0-12}$ for formoterol after $5 \mathrm{~d}$ of treatment with four inhalations of the FDC budesonide/ formoterol (160/4.5 $\mu \mathrm{g}$ ) MDI BID was 1.77-fold. ${ }^{16}$

Due to the parallel study design of PINNACLE-1, the relative bioavailability assessments of GFF MDI versus 

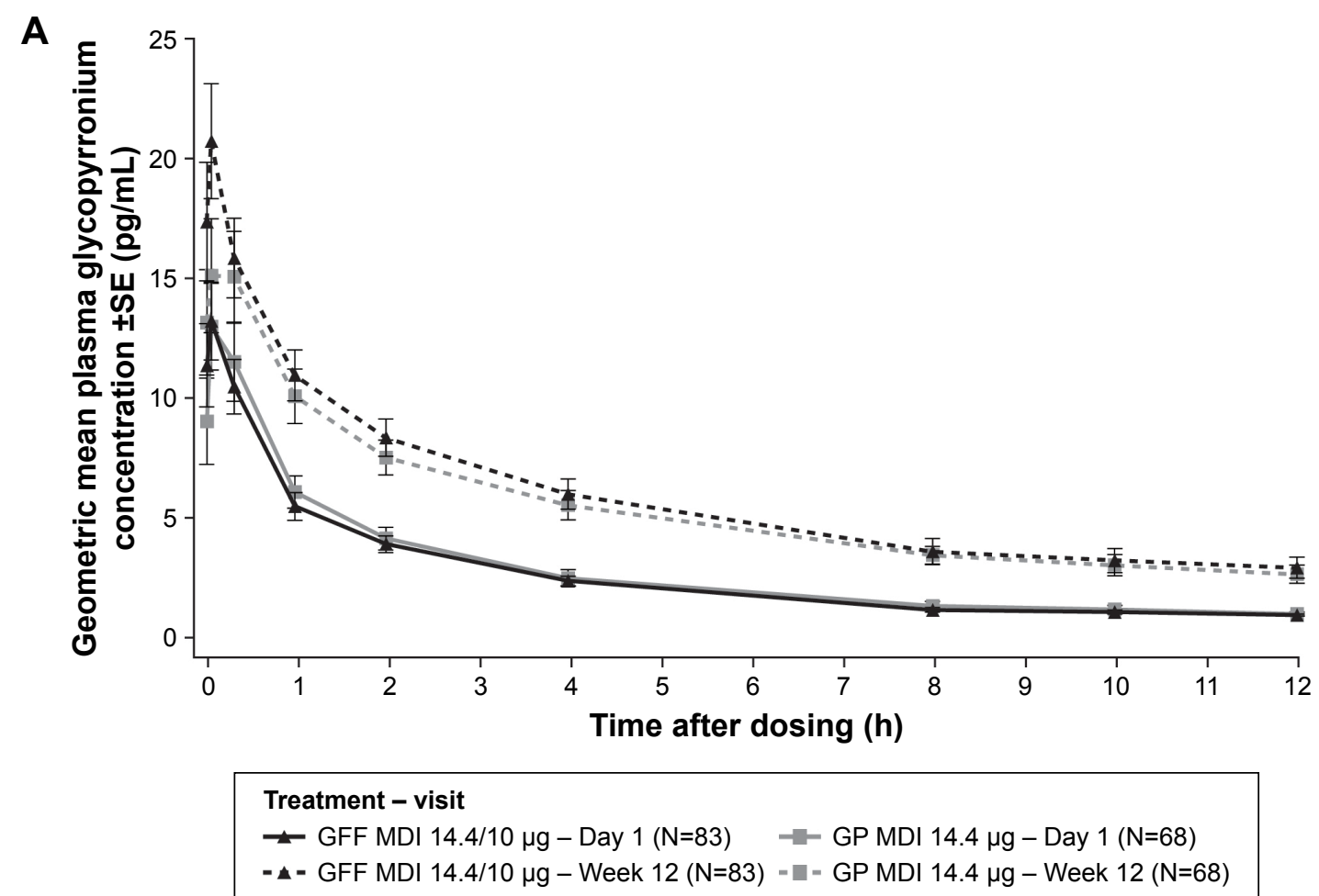

B

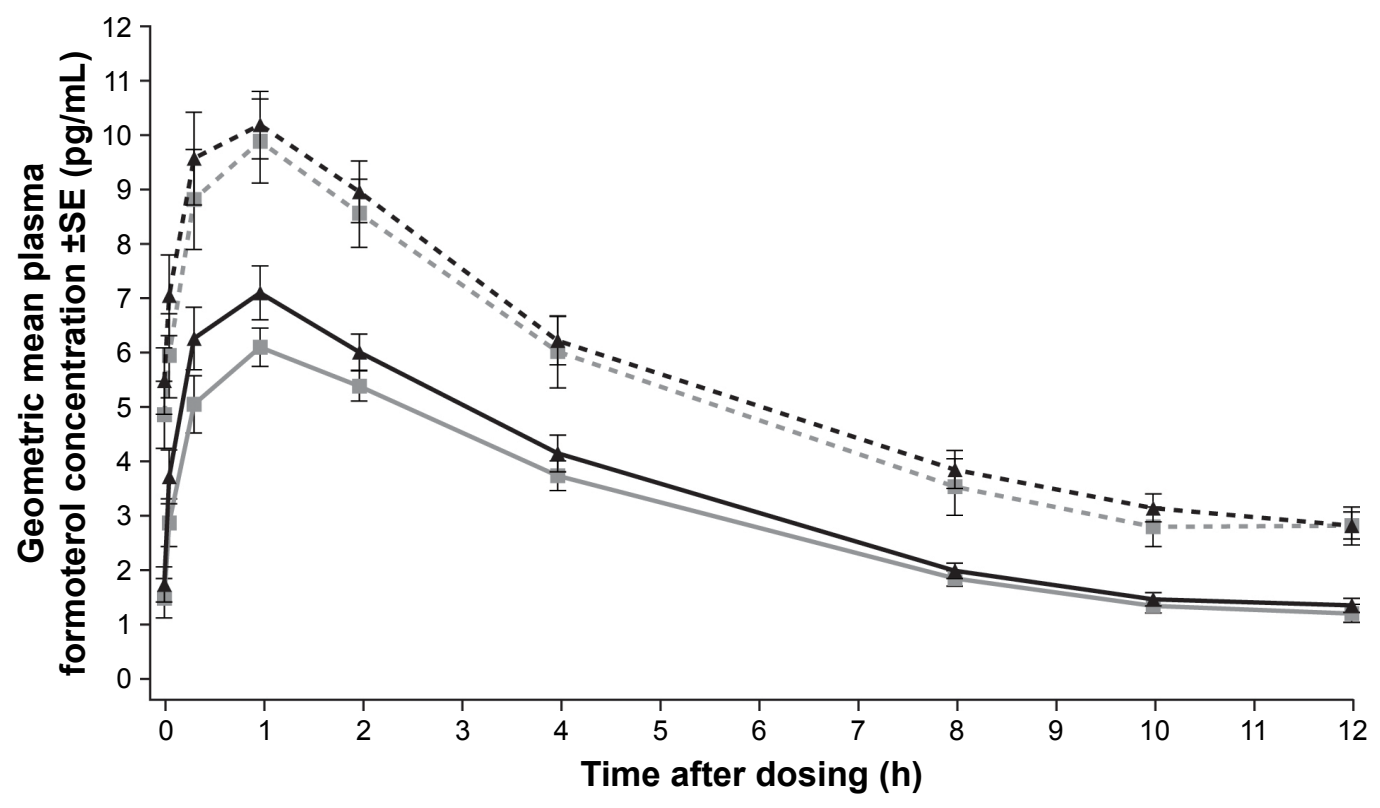

\begin{tabular}{|c|c|}
\hline Treatment - visit & \\
\hline$\leftarrow$ GFF MDI $14.4 / 10 \mu \mathrm{g}-$ Day $1(\mathrm{~N}=83)$ & $=-$ FF MDI $10 \mu \mathrm{g}-$ Day $1(\mathrm{~N}=70)$ \\
\hline - - GFF MDI 14.4/10 $\mu \mathrm{g}$ - Week $12(\mathrm{~N}=83)$ & - - FF MDI $10 \mu \mathrm{g}-$ Week $12(\mathrm{~N}=70)$ \\
\hline
\end{tabular}

Figure I Plasma concentrations after single and chronic dosing.

Notes: (A) Glycopyrronium from GFF MDI and GP MDI; (B) formoterol from GFF MDI and FF MDI (PK population).

Abbreviations: GFF, glycopyrronium/formoterol fumarate dihydrate; MDI, metered dose inhaler; GP, glycopyrronium; FF, formoterol fumarate dihydrate; PK, pharmacokinetic.

its monocomponents in this sub-study were made between subjects, rather than the within-subject evaluation that is more typical for studies purely focused on PK. Hence, the high variability observed in this study was anticipated, and bounds for equivalence valuation had not been pre-specified. However, despite the high variability at steady state (Week 12), the $90 \%$ CIs for the ratios (GFF MDI versus monocomponent MDIs) of the geometric LSMs for $\mathrm{AUC}_{0-12}$ fell within the traditional bioequivalence bounds of $80 \%$ to $125 \%$ for both glycopyrronium and formoterol, as did the $90 \%$ CI for the ratio 


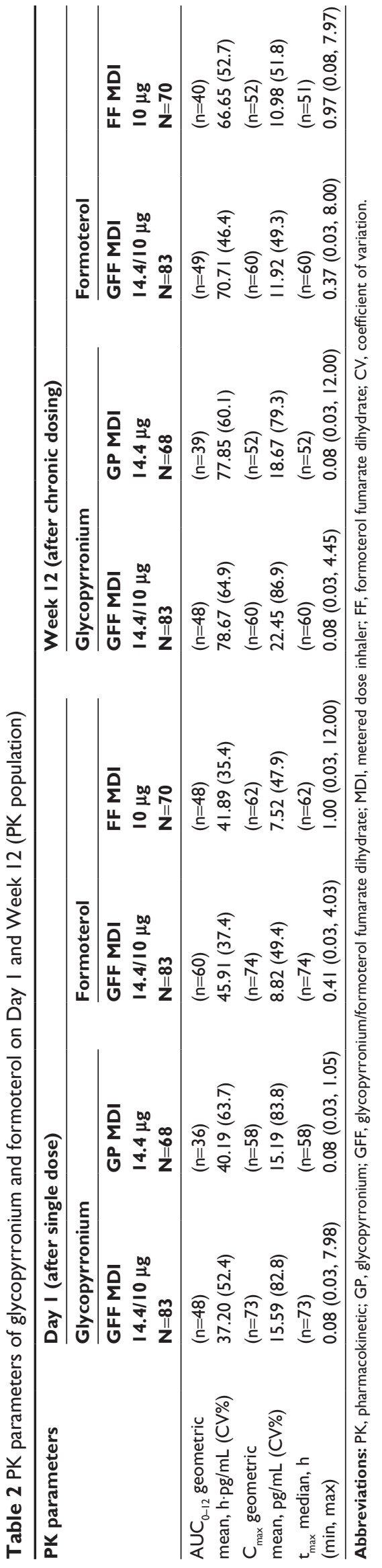

(GFF MDI versus FF MDI) of the geometric LSMs for $\mathrm{C}_{\text {max }}$ for formoterol. ${ }^{17}$ This indicated that there was no meaningful PK interaction between glycopyrronium and formoterol in patients receiving GFF MDI relative to GP MDI or FF MDI after chronic dosing (12 wks).

The median $\mathrm{t}_{\max }$ for formoterol-based individual concentration-time profiles was lower with GFF MDI compared to FF MDI ( $0.4 \mathrm{~h}$ versus $1.0 \mathrm{~h}$; Table 2$)$, although the mean $\mathrm{t}_{\max }$ for GFF MDI compared to FF MDI was comparable ( $0.9 \mathrm{~h}$ versus $1.1-1.2 \mathrm{~h}$ ). However, the study population included many elderly patients from whom it was difficult to draw blood samples, and a number of patients had samples with formoterol concentrations below the LLQ over the $12 \mathrm{~h}$ timeframe. In addition, no samples were obtained between $20 \mathrm{~min}$ and $1 \mathrm{~h}$ when the mean formoterol plasma concentration reached its peak (Figure 1B). These factors are likely to have impacted the individual concentration-time profiles, and as a result, $\mathrm{t}_{\max }$ values of $12 \mathrm{~h}$ were obtained for some patients irrespective of the analyte, which would have affected the overall median $t_{\text {max }}$ values. In the mean plasma concentration-time profiles, the time to peak formoterol plasma concentration was consistent between the formulations at Day 1 and Week 12 (Figure 1B), and therefore the difference observed based on individual concentration-time profiles was not considered to be clinically relevant.

Limitations include that in this setting of a PK substudy, dosing prior to the Week 12 assessment could not be controlled as tightly as in shorter, clinic-based PK studies; however, patients were only included in the PK population if they had not missed a dose in the $2 \mathrm{~d}$ prior to testing. Furthermore, the time to steady state for glycopyrronium and formoterol in patients receiving GFF MDI could not be estimated during this study, as the PK parameters were assessed only at Day 1 and Week 12.

Importantly, the safety and tolerability profiles of GFF MDI, GP MDI, and FF MDI have been established in patients with moderate-to-very severe COPD during the full 24 wk period of this study (PINNACLE-1). ${ }^{4}$ Of the patients treated with GFF MDI, GP MDI, FF MDI, or placebo MDI, $58.8 \%-62.9 \%$ reported at least one treatment-emergent adverse event (TEAE), and $6.4 \%-8.4 \%$ reported serious TEAEs. The incidence of cardiovascular events of special interest was low (stroke, $0 \%-0.4 \%$; cardiac ischemia, $0.4 \%-$ $2.3 \%$; cardiac failure, $1.3 \%-2.5 \%$; arrhythmia, $1.3 \%-3.2 \%$ ), and no important trends in clinical laboratory results, vital signs, or electrocardiograms were observed. ${ }^{4}$ Furthermore, these safety and tolerability results were confirmed in a similar $24 \mathrm{wk}$, randomized, controlled study (PINNACLE-2). ${ }^{4}$ 
Table 3 Accumulation ratios (Week I2/Day I) for glycopyrronium and formoterol following GFF MDI and GP MDI or FF MDI administration (PK population)

\begin{tabular}{|c|c|c|c|c|c|c|c|c|}
\hline \multirow{3}{*}{$\begin{array}{l}\text { PK } \\
\text { parameters }\end{array}$} & \multicolumn{8}{|c|}{ Glycopyrronium } \\
\hline & \multicolumn{4}{|c|}{ GFF MDI I4.4/I0 $\mu \mathrm{g}$} & \multicolumn{4}{|c|}{ GP MDI I $4.4 \mu \mathrm{g}$} \\
\hline & $\begin{array}{l}\text { n } \\
\text { Week I2/ } \\
\text { Day I }\end{array}$ & $\begin{array}{l}\text { Accumulation ratio } \\
\text { of geometric LSMa }\end{array}$ & $90 \% \mathrm{Cl}$ & $\begin{array}{l}\text { Intra-subject } \\
\text { CV\% }\end{array}$ & $\begin{array}{l}\text { n } \\
\text { Week I } 2 \text { I } \\
\text { Day I }\end{array}$ & $\begin{array}{l}\text { Accumulation ratio } \\
\text { of geometric LSMa }\end{array}$ & $90 \% \mathrm{Cl}$ & $\begin{array}{l}\text { Intra-subject } \\
\text { CV\% }\end{array}$ \\
\hline $\mathrm{AUC}_{0-12}$ & $48 / 48$ & 2.30 & $2.04,2.59$ & 30.1 & $39 / 36$ & 1.99 & $1.65,2.42$ & 48.3 \\
\hline \multirow[t]{4}{*}{$\mathrm{C}_{\text {max }}$} & $60 / 73$ & 1.40 & $1.22,1.59$ & 43.5 & $52 / 58$ & 1.26 & $1.06,1.50$ & 54.9 \\
\hline & \multicolumn{8}{|c|}{ Formoterol } \\
\hline & \multicolumn{4}{|c|}{ GFF MDI $14.4 / 10 \mu \mathrm{g}$} & \multicolumn{4}{|c|}{ FF MDI I0 $\mu \mathrm{g}$} \\
\hline & $\begin{array}{l}\mathrm{n} \\
\text { Week I2I } \\
\text { Day I }\end{array}$ & $\begin{array}{l}\text { Accumulation ratio } \\
\text { of geometric LSMa }\end{array}$ & $90 \% \mathrm{Cl}$ & $\begin{array}{l}\text { Intra-subject } \\
\text { CV\% }\end{array}$ & $\begin{array}{l}\mathrm{n} \\
\text { Week I2/ } \\
\text { Day I }\end{array}$ & $\begin{array}{l}\text { Accumulation ratio } \\
\text { of geometric LSMa }\end{array}$ & $90 \% \mathrm{Cl}$ & $\begin{array}{l}\text { Intra-subject } \\
\text { CV\% }\end{array}$ \\
\hline $\mathrm{AUC}_{0-12}$ & $49 / 60$ & 1.52 & $1.36,1.69$ & 30.3 & $40 / 48$ & 1.62 & $1.41,1.86$ & 36.7 \\
\hline$C_{\max }=-12$ & $60 / 74$ & 1.32 & $1.21,1.43$ & 26.4 & $52 / 62$ & 1.51 & $1.34,1.7 \mid$ & 38.1 \\
\hline
\end{tabular}

A 28 wk extension of both studies confirmed the long-term safety of the study treatments over $52 \mathrm{wks}$ (PINNACLE-3). ${ }^{13}$

Overall, our results consolidate similar findings of a Phase IIb study that evaluated the PK of a higher dose of GFF MDI $(28.8 / 10 \mu \mathrm{g})$ versus monocomponent MDIs after a $7 \mathrm{~d}$ treatment period, and found no significant PK interactions for glycopyrronium and formoterol when delivered as an FDC MDI using co-suspension delivery technology. ${ }^{14}$

\section{Conclusion}

The FDC GFF MDI and the individual components GP MDI and FF MDI were well tolerated and there were no safety concerns. ${ }^{4,13}$ In this $\mathrm{PK}$ sub-study, the $\mathrm{AUC}_{0-12}$ accumulation of glycopyrronium delivered from GFF MDI and GP MDI was 2.30-fold and 1.99-fold, respectively, and the $\mathrm{AUC}_{0-12}$ accumulation of formoterol delivered from GFF MDI and FF MDI was 1.52-fold and 1.62-fold, respectively,

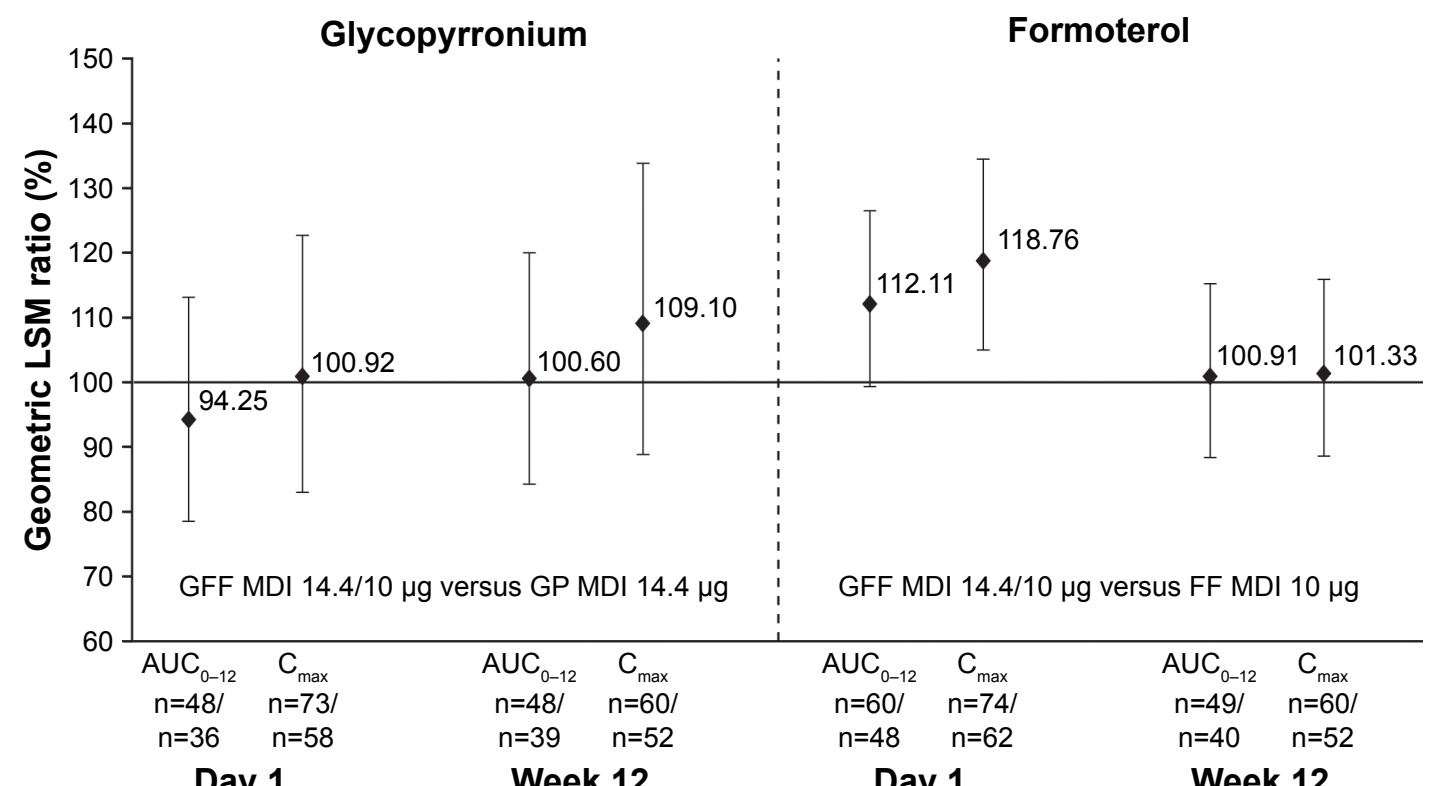

Figure 2 Relative bioavailability for glycopyrronium and formoterol following GFF MDI and GP MDI or FF MDI administration (GFF MDI/monocomponent MDI) (PK population).

Note: Vertical bars are $90 \% \mathrm{Cl}$ of the ratio (GFF MDI/monocomponent MDI) of geometric LSM.

Abbreviations: GFF, glycopyrronium/formoterol fumarate dihydrate; MDI, metered dose inhaler; GP, glycopyrronium; FF, formoterol fumarate dihydrate; PK, pharmacokinetic; LSM, least squares mean. 
after $12 \mathrm{wks}$ of treatment. The systemic exposure $\left(\mathrm{AUC}_{0-12}\right.$ and $\mathrm{C}_{\max }$ ) of glycopyrronium and formoterol was similar when delivered in the FDC GFF MDI compared to when the individual components were delivered in separate inhalers (GP MDI and FF MDI) after single and chronic dosing (12 wks), indicating that there were no meaningful PK interactions, whether drug-drug or due to formulation, between glycopyrronium and formoterol when administered in the FDC GFF MDI with co-suspension delivery technology.

\section{Acknowledgments}

The authors thank all the patients and their families and the team of investigators, research nurses, and operation staff involved in PINNACLE-1. The authors would also like to thank Chad Orevillo, Patrick Darken, Earl St Rose, and Michael Gillen for their valuable contribution to this work, and Everest Clinical Research, who performed the statistical analyses for this study. Medical writing support, under the direction of the authors, was provided by Pauline Craig, $\mathrm{PhD}$, of CMC CONNECT, a division of Complete Medical Communications Ltd, Glasgow, UK, funded by AstraZeneca, Cambridge, UK in accordance with Good Publication Practice (GPP3) guidelines. ${ }^{18}$ This study was supported by Pearl - A member of the AstraZeneca Group, and has been published in manuscript form (Martinez et al. Efficacy and Safety of Glycopyrrolate/Formoterol Metered Dose Inhaler Formulated Using Co-Suspension Delivery Technology in Patients With COPD. Chest. 2017;151(2):340-357); data from the pharmacokinetic sub-study were not included in the manuscript and have not been presented previously at any congresses.

\section{Author contributions}

All authors contributed toward conception and design, data acquisition, or data analysis and interpretation, critical revision, and providing final approval of the manuscript, and agree to be accountable for all aspects of the work.

\section{Disclosure}

GTF reports grants and personal fees from AstraZeneca, Boehringer Ingelheim, Novartis, Pearl - A member of the AstraZeneca Group, Sunovion, and Theravance. He has also received grants from Forest and personal fees from GlaxoSmithKline, Meda, Mylan, and Verona. RRR has received personal fees from AstraZeneca, Boehringer Ingelheim, Pearl - A member of the AstraZeneca Group, TEVA, Menarini, and Novartis; and grants/research support from Menarini. CR is an employee of AstraZeneca and Chief
Executive Officer of Pearl - A member of the AstraZeneca Group. AM is an employee of Pearl - A member of the AstraZeneca Group. SS and UJM are employees of AstraZeneca. The authors report no other conflicts of interest in this work.

\section{References}

1. Global Initiative for Chronic Obstructive Lung Disease. Global Strategy for the Diagnosis, Management and Prevention of COPD [updated 2018]. Available from: http://www.goldcopd.org. Accessed February 12, 2018.

2. Bateman ED, Ferguson GT, Barnes N, et al. Dual bronchodilation with QVA149 versus single bronchodilator therapy: the SHINE study. Eur Respir J. 2013;42(6):1484-1494.

3. Celli B, Crater G, Kilbride S, et al. Once-daily umeclidinium/vilanterol $125 / 25 \mu \mathrm{g}$ therapy in COPD: a randomized, controlled study. Chest. 2014;145(5):981-991.

4. Martinez FJ, Rabe KF, Ferguson GT, et al. Efficacy and safety of glycopyrrolate/formoterol metered dose inhaler formulated using cosuspension delivery technology in patients with COPD. Chest. 2017; 151(2):340-357.

5. D'Urzo AD, Rennard SI, Kerwin EM, et al. Efficacy and safety of fixed-dose combinations of aclidinium bromide/formoterol fumarate: the 24-week, randomized, placebo-controlled AUGMENT COPD study. Respir Res. 2014;15:123.

6. Singh D, Jones PW, Bateman ED, et al. Efficacy and safety of aclidinium bromide/formoterol fumarate fixed-dose combinations compared with individual components and placebo in patients with COPD (ACLIFORM-COPD): a multicentre, randomised study. BMC Pulm Med. 2014;14:178.

7. Yu AP, Guérin A, Ponce de Leon D, et al. Clinical and economic outcomes of multiple versus single long-acting inhalers in COPD. Respir Med. 2011;105(12):1861-1871.

8. Yu AP, Guérin A, Ponce de Leon D, et al. Therapy persistence and adherence in patients with chronic obstructive pulmonary disease: multiple versus single long-acting maintenance inhalers. J Med Econ. 2011; 14(4):486-496.

9. Bevespi Aerosphere ${ }^{\mathrm{TM}}$ (glycopyrrolate and formoterol fumarate) inhalational aerosol, for oral inhalation use [prescribing information]. Wilmington: AstraZeneca Pharmaceuticals LP; 2017. Available from: http://www.azpicentral.com/bevespi/bevespi_pi.pdf. Accessed February 12, 2018.

10. Lechuga-Ballesteros D, Noga B, Vehring R, Cummings RH, Dwivedi SK. Novel cosuspension metered-dose inhalers for the combination therapy of chronic obstructive pulmonary disease and asthma. Future Med Chem. 2011;3(13):1703-1718.

11. Vehring R, Lechuga-Ballesteros D, Joshi V, Noga B, Dwivedi SK. Cosuspensions of microcrystals and engineered microparticles for uniform and efficient delivery of respiratory therapeutics from pressurized metered dose inhalers. Langmuir. 2012;28(42):15015-15023.

12. Doty A, Schroeder J, Vang K, et al. Drug delivery from an innovative LAMA/LABA co-suspension delivery technology fixed-dose combination MDI: evidence of consistency, robustness, and reliability. AAPS Pharm Sci Tech. 2018;19(2):837-844.

13. Hanania NA, Tashkin DP, Kerwin EM, et al. Long-term safety and efficacy of glycopyrrolate/formoterol metered dose inhaler using novel Co-Suspension ${ }^{\mathrm{TM}}$ Delivery Technology in patients with chronic obstructive pulmonary disease. Respir Med. 2017;126:105-115.

14. Reisner C, Fabbri LM, Kerwin EM, et al. A randomized, seven-day study to assess the efficacy and safety of a glycopyrrolate/formoterol fumarate fixed-dose combination metered dose inhaler using novel Co-Suspension ${ }^{\mathrm{TM}}$ Delivery Technology in patients with moderateto-very severe chronic obstructive pulmonary disease. Respir Res. 2017;18(1):8. 
15. Li L, Li X, Xu L, Sheng Y, Huang J, Zheng Q. Systematic evaluation of dose accumulation studies in clinical pharmacokinetics. Curr Drug Metab. 2013;14(5):605-615.

16. Eklund A, Tronde A, Johannes-Hellberg I, Gillen M, Borgström L. Pharmacokinetics of budesonide and formoterol administered via a series of single-drug and combination inhalers: four open-label, randomized, crossover studies in healthy adults. Biopharm Drug Dispos. 2008; 29(7):382-395.
17. Lu D, Lee SL, Lionberger RA, et al. International guidelines for bioequivalence of locally acting orally inhaled drug products: similarities and differences. AAPS J. 2015;17(3):546-557.

18. Battisti WP, Wager E, Baltzer L, et al. Good publication practice for communicating company-sponsored medical research: GPP3. Ann Intern Med. 2015;163(6):461-464.

International Journal of COPD

\section{Publish your work in this journal}

The International Journal of COPD is an international, peer-reviewed journal of therapeutics and pharmacology focusing on concise rapid reporting of clinical studies and reviews in COPD. Special focus is given to the pathophysiological processes underlying the disease, intervention programs, patient focused education, and self management protocols.
Dovepress

This journal is indexed on PubMed Central, MedLine and CAS. The manuscript management system is completely online and includes a very quick and fair peer-review system, which is all easy to use. Visit $\mathrm{http}: / / \mathrm{www}$.dovepress.com/testimonials.php to read real quotes from published authors.

Submit your manuscript here: http://www.dovepress.com/international-journal-of-chronic-obstructive-pulmonary-disease-journal 